\title{
Consideraciones sobre el discurso oral en el aula
}

María Elvíra Rodríguez Luna'

Docente investigadora de la Universidad Distrital Francisco José de Caldas

\section{RESUMEN}

Se propone una reflexión sobre las funciones del discurso oral en el contexto de la escuela y la incidencia de ciertas concepciones de los profesores sobre el desarrollo de la oralidad en el aula. Parte de señalar algunas referencias teóricas sobre el discurso en general y las funciones de la oralidad en particular, con el objeto de relacionarlas con los fundamentos y propósitos expresados en el currículo para el área de Lengua Castellana. A partir de varios estudios de caso realizados por los grupos de investigación referenciados en el texto, sitúa las concepciones de los profesores sobre la oralidad y las posibilidades de transformación de la enseñanza y la evaluación del discurso oral en el aula. Concluye con la formulación de preguntas pertinentes para posibles investigaciones en este campo.

Palabras clave

Oralidad, discurso oral, evaluación en lengua materna.

\section{ABSTRACT}

This article proposes a reflection on the functions of the oral speech in the context of the school and the incidence that have certain conceptions rooted in the professors on the development of this activity in the classroom. Part to indicate to some theoretical references on the speech in general and the functions of the oralidad in individual, with the intention of relating them to the foundations and intentions expressed in Curriculum for the area of Castilian Language. Based on several studies of case made by the groups of investigation mentioned in the text, it locates the conceptions of the professors on the orality and the possibilities of transformation of teaching and evaluation of the oral discourse in the classroom. It finalizes by formulating questions for possible investigations in this field.

\section{KEY WORDS}

Orality, oral speech, evaluation in maternal language.

1 Licenciada en Filología e Idiomas de la Universidad Nacional de Colombia. Magíster en Lingüística Hispánica del Seminario Andrés Bello (Instituto Caro y Cuervo), Doctora en Ciencias Pedagógicas Universidad Central de las Villas. Profesora Titular de la Especialización en Lenguaje y Pedagogía de Proyectos de la Universidad Distrital. Miembro de los Grupos de Investigación Lenguaje, Identidad y Cultura de la Universidad Distrital y Evaluación de la Universidad Nacional de Colombia (Clasificados en Categoría A de Colciencias). 


\section{Introducción}

$\mathrm{L}$ a presente reflexión está centrada en el discurso oral en el aula y la manera como se orienta y evalúa esta actividad del lenguaje en el contexto escolar. Con tal propósito se presentan algunos elementos -más como interrogantes que como respuestas acabadas y concluyentescon el objeto de destacar de qué modo se asume, casi de manera generalizada, el objetivo de fortalecer la competencia oral (hablar-escuchar) en el aula, el lugar que se le concede en el currículo, las necesidades existentes frente a una evaluación que asuma el conjunto de las actividades del lenguaje (hablar, escuchar, leer, escribir) y las perspectivas investigativas que surgen del vacío existente respecto al estudio y abordaje de este tipo de problemas en nuestro medio.

Dada su complejidad y los diferentes aspectos que entran en juego para la comprensión y búsqueda de alternativas orientadas a la solución de este problema, solamente se presentan algunos planteamientos derivados de las experien-. cias de formación y de investigación realizadas por los grupos de investigación Lenguaje, Identidad y Cultura de la Universidad Distrital y el Grupo de Evaluación de la Universidad Nacional de Colombia. En cuanto a la manera como se asume por parte de los profesores el sentido de lo oral y su evaluación en el aula, se toman como referencias: el resultado de una encuesta aplicada a docentes de la Educación Básica y Media de Bogotá y los trabajos de campo realizados por un grupo de estudiantes del Semillero de Investigadores en Evaluación de la Universidad Distrital ${ }^{2}$.

\footnotetext{
2 Se hace referencia a las monografías de grado tituladas "Política y realidad: un análisis sobre la evaluación interna en lengua castellana" (realizada por Mónica Viviana González Castillo y Lidia Bibiana Pinto Millán) y "Qué pensamos cuando evaluamos: la evaluación al tablero" (elaborada por Paola Andrea Herrera Morales, Paola Andrea Gamez Linares, Angela Torres García, Jenny Carolina Corredor y José Francisco Quintero Monroy). Estos trabajos se desarrollan en el marco del proyecto interinstitucional "Construcción de una red nacional de investigación en evaluación", liderada por el Grupo de Evaluación de la Universidad Nacional de Colombia con la participación de la Universidad Distrital y Pedagógica, el apoyo del ICFES y el aval de Colciencias.
}

Como lo indica el título del artículo, se trata de una formulación preliminar sobre el tema, en la perspectiva de iniciar una exploración sobre un campo de gran interés para la investigación en lengua materna.

\section{Consideraciones.iniciales}

\section{Sobre el discurso oral}

Para comprender la naturaleza del discurso oral, partimos de considerarlo como una práctica social que implica formas de acción entre las personas y que se articula a partir del uso lingüístico contextualizado. En cuanto práctica social, el discurso se materializa en el uso de determinada lengua con fines específicos ligados a su contexto de realización (lingüístico, cognitivo, sociocultural), bien sèa a través de la oralidad o de la escritura.

En consecuencia, la modalidad escrita y la oralidad son prácticas sociales que integran diversas dimensiones de la actividad humana, dentro de las cuales destacamos la cognitiva, la intersubjetiva y la cultural. Asumimos que-la lengua constituye el material inicial (fónico, gráfico, morfosintáctico, léxico) del que disponen los miembros de una comunidad para elegir/decidir de qué modo interactuar discursivamente. Tal como lo señalan Calsamiglia y Tusón: "Las lenguas viven en el discurso y a través de él" (1999). Dicha elección se realiza según parámetros comunicativos que incluyen la situación de comunicación, el contexto de la situación, los propósitos de quien la realiza, las características de los destinatarios, entre otros.

Recordemos que para la descripción del discurso oral Hymes (1967) propuso el modelo SPEAKING (situación, participantes, finalidades, secuencia de actos, clave, instrumentos, normas y género) en el cual incorpora los elementos propios de un evento comunicativo. Tales parámetros son de orden cognitivo y sociocultural, son cambiantes y posibilitan la comprensión de 
cómo se despliegan en cada cultura las actividades de comunicación. En consecuencia, la competencia comunicativa propuesta por Hymes (1972: 32) apunta hacia la determinación de dimensiones como: "las capacidades de las personas, la organización de los medios verbales para propósitos socialmente definidos, y las reglas sensitivas a las situaciones".

Por consiguiente, aunque el lenguaje se materializa a través de la oralidad o la escritura y. éstas comparten algunos rasgos, también tienen diferencias significativas, a pesar de ser ambas expresión de la facultad humana del lenguaje. Por una parte, la oralidad es natural, consustancial al ser humano y constitutiva de la persona como miembro de la especie; por otra, la escritura no es universal e implica el dominio de una tecnología (Ong, 1987).

Pero no todas las manifestaciones de la oralidad son "naturales", pues si bien muchas de sus funciones se adquieren a través del uso cotidiano de la lengua, existen otras formas que requieren una orientación particular. Así, por ejemplo, una conferencia, una alocución presidencial, un discurso inaugural, una exposición, una sustentación, entre otras, exigen un alto grado de preparación, de elaboración, y casi siempre incorporan el soporte de la escritura (un guión, un cuadro sinóptico, unas notas, una diapositiva; etc.). De allí que los profesores de lengua debamos contar con el fundamento investigativo, pedagógico y didáctico adecuado a efecto de desarrollar plenamente todas las funciones de la oralidad como parte de la educación lingüística.

Sin embargo, como lo plantea Reizábal (1993: 76): "Por desconocimiento o por desinterés, muchos docentes no "enseñan a hablar ni a escuchar"; creen que con proponer o permitir algunos debates o exposiciones en clase y corregir ciertos errores articulatorios, morfológicos, sintácticos o semánticos, ya han cumplido con los objetivos de la comunicación oral. Otros se obsesionan por las correcciones lingüísticas, olvidando que la competencia comunicativa implica mucho más que conocer y manejar la norma, ya que mediante el lenguaje se transmite no sólo lo que sabemos, sino lo que somos, pues se hace patente la persona entera". La situación en nuestro contexto no es muy diferente.

\section{Algunas de las funciones de la oralidad}

En tanto modalidad del lenguaje, la oralidad desarrolla las funciones cognitiva, interactiva y estética del lenguaje. Dentro de ellas, la función esencial del discurso oral, como ya lo señalamos al comienzo, es la de construir las relaciones sociales, tanto en la vida cotidiana, como en la actividad institucional y en los espacios lúdicos.

- Función interactiva. En la vida cotidiana, la oralidad cumple la función esencial de construir y mantener las relaciones entre los miembros de una comunidad. En algunas sociedades el discurso oral constituye la única modalidad a disposición de los usuarios de la.lengua. Por eso, es evidente que cuando rompemos las relaciones con otros "les dejamos de hablar". El silencio, la ruptura de la comunicación oral implica la pérdida de los nexos afectivós, amorosos, familiares, sociales, culturales. No hablamos cuando nos sentimos en un ambiente hostil, cuando no compartimos los parámetros culturales de la situación, cuando no sabemos de qué modo hablar, de qué hablar, para qué hablar, cuando de antemano consideramos que nuestra voz no será escuchada porque no nos sentimos como sujetos de lenguaje.

Por lo tanto, también en la vida cotidiana la apropiación de las formas adecuadas del discurso oral -según las situaciones que se nos presentan y según el conocimiento de la cultura respecto a lo adecuado o inadecuado del habla y de sus formas-, constituye un aprendizaje. El discurso oral posibilita así acceder a la cultura, comprenderla y actuar en consecuencia.

En el dominio de lo público, de lo institucional, la oralidad tiene también un lugar importante. Así por ejemplo, en el campo de la política quien domina el discurso oral ocupa un lugar destacado entre su comunidad y puede acceder con mayor facilidad a cargos de elección; en el campo del derecho, con la incorporación de las audiencias orales, se requiere cada vez más el dominio de una competencia oral por parte de quienes actúan 
como abogados. En el aula, es indudable que le prestamos mayor atención a quien es capaz de pedir la palabra, de intervenir en la clase, de exponer sus ideas con mayor fluidez.

Esto para solamente mencionar algunos de los ámbitos de la actividad social en los que el discurso oral cumple una función importante de poder y dominación. Por esto se considera que cuanto más democrática sea una sociedad, mayores espacios deberá brindar para la expresión de la palabra.

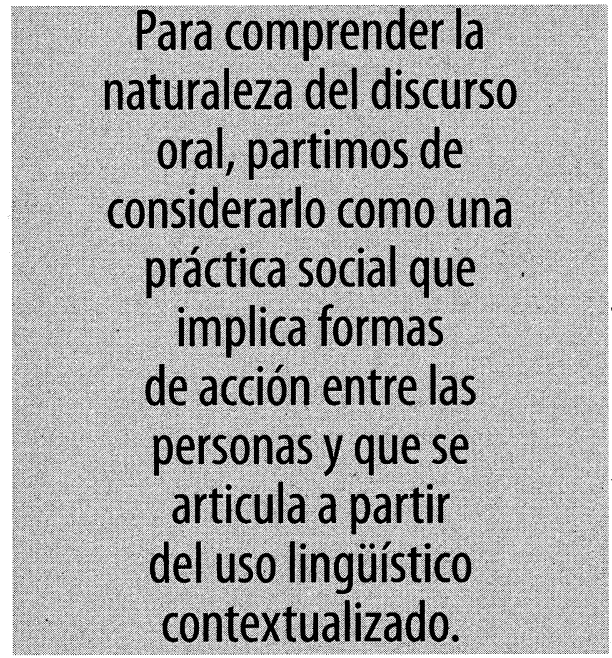

De otra parte, "la oralidad secundaria" proporcionada por los medios de comunicación de masas, constituye también una función importante en las sociedades actuales para la cohesión, el debate y la información. De este modo, las entrevistas, las propagandas radiales, los noticieros, entre otras manifestaciones del discurso oral, posibilitan la construcción de tejido social, del sentido de pertenencia a una colectividad, de la consecución de metas comunes; como también la generación de espacios para la divergencia, el conflicto o la pluralidad.

- Función estética. En el campo de la estética y la lúdica, la oralidad también ocupa un lugar destacado: los mitos, las leyendas, las coplas, los refranes, los cuentos tradicionales, los chistes, el teatro, entre otras formas de recreación de la realidad, tienen como material básico la oralidad. Los niños desarrollan tempranamente juegos del lenguaje que les posibilitan la apropiación de la lengua materna al igual que su ingreso a la cultura. Es ésta una de las funciones a las cuales se confiere un lugar importante en el currículo de la educación preescolar y básica primaria. Sin embargo, la mayoría de las veces tan sólo se asumen como temas que requieren ser tratados y en pocas oportunidades se desarrolla un trabajo sistemático sobre la apropiación de los formatos y su transposición a nuevas situaciones de comunicación.

- Función cognitiva. Halliday (1986), destaca dentro de las funciones del lenguaje del niño, además de la instrumental ("Quiero"): para satisfacer necesidades materiales; la reguladora ("Haz lo que te digo"): para regular el comportamiento de los demás; la interactiva ("Yo y tú"): para involucrar a otras personas; la personal ("Aquí estoy"): para identificar y manifestar el yo; la imaginativa ("Finjamos"): para crear un mundo propio; la informativa ("Tengo algo que decirte"): para comunicar nuevos informes, una función muy importante en el plano cognitivo. Se trata de la función heurística ("Dime por qué"): para explorar el mundo exterior e interior." En este sentido es importante destacar la función que cumple la pregunta no sólo en la etapa inicial sino a lo largo de la experiencia escolar para la apropiación y construcción de conocimientos con los otros, a partir de la interacción y el procesamiento de la información.

De otra parte, es necesario resaltar el papel de la oralidad en la planificación de las acciones y la organización del pensamiento, el planteamiento y verificación de hipótesis, el esclarecimiento de dudas, entre otras importantes funciones ligadas al desarrollo del pensamiento y el conocimiento, como actividades básicas de la enseñanza en la escuela.

Reconocida la importancia de la oralidad y la necesidad de asumirla como objeto de enseñanza, es importante relacionar las funciones anteriormente señaladas con el desarrollo de las competencias básicas, teniendo presente el lugar que se le concede en el currículo. 


\section{Planteamientos sobre la oralidad en el currículo}

\section{Los lineamientos curriculares}

En los lineamientos curriculares se propone abordar las actividades de hablar, escuchar, leer y escribir en función de la significación y la producción de sentido. De manera particular, en cuanto a la oralidad se plantea que:

Escuchar, por ejemplo, tiene que ver con elementos pragmáticos como el reconocimiento de la intención del hablante, el reconocimiento del contexto social, cultural, ideológico, desde el cual se habla; además, está asociado a complejos procesos cognitivos, ya que a diferencia del acto de leer en el que se cuenta con el impreso como soporte de la comunicación, escuchar implica ir tejiendo el significado de manera inmediata, con pocas posibilidades de volver atrás en el proceso interpretativo de los significados. A su vez, hablar resulta ser un proceso igualmente complejo, es necesario elegir una posición de enunciàción pertinente a la intención que se persigue, es necesario reconocer quién es el interlocutor para elegir un registro de lenguaje y un léxico determinado, etc. (MEN, 1998a: 50 - 51).

También se señala que estas actividades, al igual que la lectura y la escritura, requieren el desarrollo de competencias asociadas a:

- Una competencia gramatical o sintáctica, referida a las reglas sintácticas, morfológicas, fonológicas y fonéticas que rigen la producción de los enunciados lingüísticos:

- Una competencia textual referida a los mecanismos que garantizan coherencia y cohesión a los enunciados (nivel micro) y a los textos (nivel macro). Esta competencia está asociada, también, con el aspecto estructural del discurso, jerarquías semánticas de los enunciados, uso de los conectores, por ejemplo; y con la posibilidad de reconocer o seleccionar según las prioridades e intencionalidades discursivas, diferentes tipos de textos.
- Una competencia semántica referida a la capacidad de reconocer y usar los significados y el léxico de manera pertinente según las exigencias del contexto de comunicación. Aspectos como el reconocimiento de campos semánticos, tecnolectos o idiolectos particulares hacen parte de este competencia; lo mismo que el seguimiento de un eje o hilo temático en la producción discursiva.

- Una competencia pragmática o sociocultural referida al reconocimiento y al uso de las reglas contextuales de la comunicación. Aspectos como el reconocimiento de intencionalidades y variables del contexto como el componente ideológico y político que está detrás de los enunciados hacen parte de esta competencia, el reconocimiento de variaciones dialectales, registros diversos o, en términos de Bernstein, códigos sociolingüísticos, presentes en los actos comunicativos son también elementos de esta competencia.

- Una competencia enciclopédica, referida a la capacidad de poner en juego los saberes con los que cuentan los sujetos y que son construidos en el ámbito de la cultura escolar o

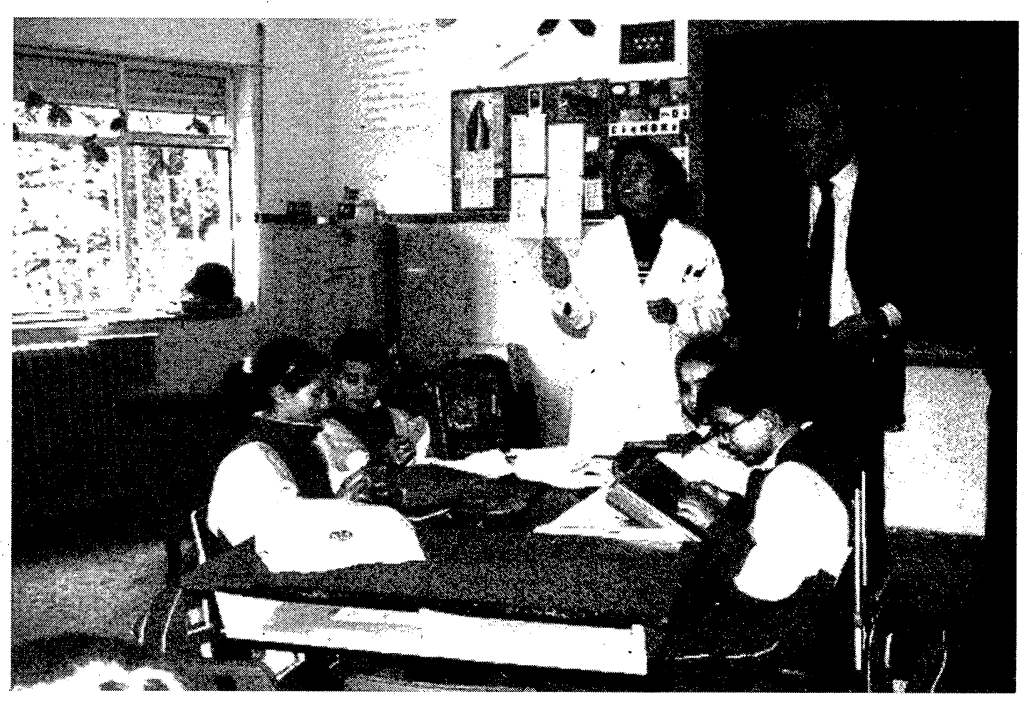

sociocultural en general, y en el microentorno local y familiar.

- Una competencia literaria entendida como la capacidad de poner en juego un saber surgido de la experiencia de lectura y análisis de las obras mismas. 
- Una competencia poética, entendida como la capacidad del sujeto para inventar mundos posibles a través de lenguajes en el uso de los mismos... tiene que ver con la búsqueda de un estilo personal."(Ibid.)

Lo anterior significa que desde el plano institucional y normativo, se han planteado las orientaciones para el desarrollo de la oralidad en el contexto de la escuela. Sin embargo, al revisar estos lineamientos, surgen preguntas como:

\section{El discurso oral posibilita así acceder a la cultura, comprenderla y actuar en consecuencia.}

¿Efectivamente, trabajamos esțas 'competencias en la oralidad?

¿Cómo las orientamos para promover el desarrollo de la oralidad en el aula?

¿Qué aspectos gramaticales trabajamos de manera sistemática?

¿Analizamos la estructura de los distintos textos orales?

¿A partir de qué modelo discursivo?

¿Reconocemos la relación existente entre los discursos orales y los contextos en los cuales se producen?

¿Proponemos estrategias adecuadas para asumir el análisis del discurso oral en todas sus dimensiones?

¿Evaluamos la producción oral? ¿Qué evaluamos? ¿Para qué evaluamos?

Los espacios escolares estudiados por el Grupo de Investigación indican que el trabajo sistemático sobre el discurso oral en el aula es escaso y fragmentado. Los propios lineamientos curriculares ponen su énfasis en la lectura y la escritura pero aportan pocos elementos explícitos para el tratamiento de los discursos orales como prácticas sociales.

\section{Los estándares curriculares}

Por su parte, también los Estándares de Lengua Castellana proponen: "un acercamiento a la pedagogia de la lengua castellana, que contemple no sólo sus características formales sino, y ante todo, sus características como sistema simbólico y sus implicaciones en los órdenes cognitivo, pragmático, emocional, cultural e ideológico, en los diferentes interlocutores del proceso de comunicación pedagógica".

De esta manera será posible desarrollar cabalmente las competencias que permiten comunicar, conocer e interactuar con la sociedad y con los otros, pues, como lo plantea van Dijk: "Muy pocos aspectos del discurso (sentido, coherencia, acción, etc.) pueden comprenderse y explicarse como corresponde, sin remitirnos a la mente de los usuarios del lenguaje verbal. Además de los recuerdos y experiencias personales de sucesos (modelos), las representaciones socioculturales compartidas (conocimientos, actitudes, ideologías, normas, valores) [...] también desempeñan un papel fundamental en el discurso, así como en su descripción y explicación)" (2003).

Lo anterior indica que, desde el punto de vista de los Lineamientos Curriculares y los Estándares, esto es, "del deber ser", están dados los elementos para desarrollar las competencias adecuadas tanto en el plano de lo oral como de lo escrito. Sin embargo, los trabajos de campo adelantados muestran que las estrategias pedagógicas se centran preferencialmente en la lectura y la escritura, descuidando el trabajo sistemático sobre el discurso oral.

Esto tiene que ver con ciertas creencias de los profesores sobre la oralidad que los lleva a considerar muchas veces que su desarrollo es natural o espontáneo y que no se requiere dispositivos pedagógicos especiales para apoyarla.

\section{Algunas creencias de los profesores con respecto a la oralidad}

\section{Lo que dicen los profesores con respecto a la oralidad}

Muchas de las creencias de los profesores respecto a la oralidad se expresan del siguiente modo:

a. La oralidad es inherente al desarrollo humano y, por lo tanto, no exige una enseñanza sistemática. 
b. La oralidad es un medio para comunicarse, pero no un objeto de estudio y enseñanza particular.

c. En lo oral, lo esencial es garantizar el buen uso de la lengua, entendiendo como tal la corrección la fonética o la norma culta en cuanto al léxico, al respecto dicen: "hablar bien ẹ hablar correctamente", "pronunciar bien", "no utilizar palabras vulgares, inadecuadas, propias de gente inculta".

d. La oralidad no tiene el mismo estatus. cultural que la escritura; escribir es difícil, su dominio confiere prestigio, el habla o la escucha no requieren mayor esfuerzo.

e. La oralidad sirve para evaluar la comprensión de lectura o los conocimientos gramaticales, pero no constituye un objeto de enseñanza en sí mismo.

f. El desarrollo de la oralidad es un problema de expertos: locutores, políticos.

g. La oralidad no requiere los procesos metacognitivos, ni metalingüísticos que se desarrollan frente a la lectura o la escritura "porque no es tan compleja ni tan importante”.

Estas creencias respecto a la importancia de la oralidad y sus manifestaciones en el aula fueron corroboradas en una encuesta aplicada a 68 docentes de la Educación Básica y Media, en la que se incluyó la siguiente pregunta: ¿Realiza evaluaciones sobre las producciones orales de los estudiantes? ¿Cómo? ¿Qué aspectos tiene en cuenta?33.

Los resultados obtenidos en 16 de las 68 respuestas, es decir, el porcentaje más alto de la muestra (equivalente al 24\%), los profesores reconocen abiertamente que este aspecto de la competencia comunicativa está ausente de la evaluación porque evidentemente el discurso oral no constituye un objeto de enseñanza específico.

En los restantes profesores (casos en los cuales se afirma hacer evaluación sobre la oralidad), los criterios se orientan hacia aspectos de orden fonético, tales como la pronunciación, el volumen, el timbre (22\%); en menor proporción se consideran importantes las categorías relativas al

\footnotetext{
${ }^{3}$ Se hace referencia a docentes que cursaron el PFPD en Pedagogía de Proyectos y Didáctica de la Lengua $\mathrm{Ma}$ terna de la Universidad Distrital de Bogotá, 2005.
}

componente semántico visto desde el plano local (vocabulario y muletillas, $12 \%$ ); aspectos semánticos de carácter global (claridad y coherencia, 16\%) mientras que las consideraciones pragmáticas, relativas a la adecuación a las situaciones de comunicación, auditorio, etc., se reducen a un $10 \%$.

Este resultado es explicable por el predominio de la concepción de la evaluación como verificación y control. Por eso, la muestra arrojó un alto porcentaje de respuestas que reducen el problema de la oralidad a la constatación de cuánta información se retiene por parte de los alumnos frente a una lectura, la cual se puede determinar a partir de las preguntas de tipo oral. Esto indica la existencia de una confusión entre el desarrollo de la competencia (oralidad) y el tipo de pruebas empleadas (orales o escritas).

Las respuestas dadas fueron:

No hay evaluación de la producción oral $16 / 68(24 \%)$

Se hace énfasis en la pronunciación, entonación y volumen 15/68 (22\%)

Se evalúa la claridad y coherencia $11 / 68$ $(16 \%)$

Se hace la verificación de la retención de información 11/68 (16\%)

Se determina la utilización de vocabulario, muletillas $8 / 68$ (12\%)

Se pondera el uso de ayudas y material $4 / 68(6 \%)$

Se evalúa la adecuación al auditorio $3 / 68$ (4\%)

Estos resultados también corroboran algunas de las conclusiones derivadas por la Colegiatura de Lenguaje de Icfes (2005), respecto a la necesidad de incorporar la oralidad como objeto de la evaluación interna y externa, teniendo en cuenta que la adopción del enfoque por competencias y/o habilidades para la vida, supone entre otros propósitos "usar información escrita y oral necesaria para la participación solidaria, crítica y creativa del ciudadano en la sociedad, apropiarse de información significativa para el propio desarrollo y la autonomía y aportar al desarrollo de la sociedad mediante las propias capacidades"(Atorresi, 2005).

Aunque en el caso del lenguaje las competencias que el usuario de una lengua requiere do- 
minar para poder comunicarse eficazmente son las de hablar, escuchar, leer y escribir, dadas las condiciones de las evaluaciones masivas, es explicable mas no lo deseable, que éstas con frecuencia se limiten al campo de la lectura debido a dificultades inherentes a la validez y confiabilidad de los resultados frente a las otras actividades. Sin embargo, existen experiencias significativas en cuanto a la evaluación de lo oral, tales como la evaluación en lenguas extranjeras y algunas pruebas masivas aplicadas en Argentina, que valdría la pena explorar para buscar caminos conducentes al mejoramiento de estos procesos evaluativos en el aula.

Es necesario resaltar el papel de
la oralidad en la planificación de
las acciones y la organización del
pensamiento, el planteamiento
y verificación de hipótesis, el
esclarecimiento de dudas, entre
otras importantes funciones
ligadas al desarrollo del
pensamiento y el conocimiento,
como actividades básicas de la
enseñanza en la escuela.

\section{Lo que se enseña de oralidad}

Como consecuencia de estas concepciones o creencias, respecto a la oralidad, en los espacios escolares con frecuencia:

a. Se enseñan las variedades dialectales para ilustrar las diferencias sociales, regionales o individuales, pero no se analizan las implicaciones culturales e ideológicas derivadas del uso de determinados registros.

b. Se enseñan las formas correctas de la lengua, sin tener en cuenta el contexto de producción del discurso oral, pero poco se reflexiona sobre el papel de la oralidad en contextos cotidianos de la comunicación, donde lo inçorrecto gramatical o normativamente puede resultar lo más apropiado de acuerdo con la situación. c. Se enseñan los elementos de la comunicación oral, pero no cómo analizar la pertinencia de los discursos orales respecto a la situación en la que se producen.

d. Se enseñan los parámetros de la comunicación oral y la identificación de sus componentes, pero no las estrategias discursivas que se ponen en juego para lograr los propósitos de la comunicación según la situación.

e. Cuando la lectura se constituye en soporte del discurso oral, se enseña a identificar los propósitos en la lectura, pero no los matices ideológicos implicados en el discurso oral y los efectos en los interlocutores. .

f. Se enseñan las características de los destinatarios de los discursos "formales" (en abstracto), pero no su puesta en escena en situaciones reales de comunicación.

g. Se enseña la definición de los tipos de discursos, pero no se trabaja en la organización textual de los diversos géneros discursivos orales.

h. Se enseña el papel de la escucha, pero no los problemas relacionados con el procesamiento de la escucha por parte de los estudiantes, a pesar de que se sabe que el $80 \%$ de la actividad en el aula es "escucha" para el estudiante.

i. Se enseña la definición de los géneros orales: radionovelas, narraciones orales tradicionales, entrevistas, pero no se aborda la comprensión oral de los mismos; por ejemplo, caracterización de las voces de los personajes, matices de significación a través de la voz, estructuración textual, discursos que circulan en estos textos, valoraciones ideológicas, posicionamientos críticos frente a estos géneros.

En algunas experiencias de campo llevadas a cabo por estudiantes de la Licenciatura en Lengua Castellana que hacen parte del.Semillero de Investigación en.Evaluación de la Universidad Distrital, se corrobora el predominio de una evaluación de la oralidad, centrada en ${ }^{4}$ :

4 Trabajos de grado titulados "Política y realidad: un análisis sobre la evaluación interna en Lengua Castellana" y "Qué pensamos cuando evaluamos: la evaluación al tablero". 
a. la pronunciación, las pausas, el léxico correcto (fonético/sintáctico/morfológico) especialmente a partir de la lectura en voz alta.

b. las ayudas audiovisuales como apoyo a lo oral (carteleras, imágenes, cuadros sinópticos).

c. Los aspectos kinésicos y proxémicos de modo normativo. De allí recomendaciones como: "hay que pararse derecho, mirar al auditorio, no mascar chicle, llevar la ropa adecuada, no pasarse la mano por la cara", etc.

d. Las definiciones: ¿qué es una mesa redonda? ¿En qué consiste un debate? ¿Cuáles son los elementos de la comunicación oral? ¿Cuáles son las características de un buen orador?

En síntesis, en la escuela muchas veces olvidamos que: "no sólo "conocemos" nuestra lengua materna como un sistema abstracto de signos vocales, o como si fuera una especie de texto de gramática con un diccionario adjunto; la conocemos en el sentido de saber cómo utilizarla, sabemos cómo comunicarnos con otras personas, cómo elegir formas de lenguaje apropiadas al tipo de situación en que nos encontramos... sabemos comportarnos lingüísticamente" (Halliday, 1986:23).

Por eso, aunque la producción oral se constituye en una de las competencias básicas abordadas en todos los niveles de la escolaridad, sigue siendo un campo sobre el cual se necesita trabajar de manera más sistemática y profunda.

\section{Perspectivas para el tratamiento significativo de la oralidad en el aula}

A pesar de las orientaciones dadas a la oralidad en el aula, según las situaciones anteriormente presentadas, es importante retomar algunas propuestas especialmente encaminadas al desarrollo de la oralidad en nuestro contexto educativo cuyos resultados muestran avances significativos en el campo pedagógico y didáctico. Al respecto, se mencionará el desarrollo de tres proyectos de investigación por parte del Grupo de Investigación Lenguaje, Identidad y Cultura ${ }^{5}$.

\footnotetext{
5 Se hace referencia, en su orden, a los siguientes proyectos de investigación: 1 . "Conocimiento del mundo, conocimiento lingüístico y entorno sociocultural en la adquisi-
}

\section{Proyectos de investigación centrados en oralidad}

En el primero de los proyectos enunciados se resalta la sensibilización del docente hacia las actividades del lenguaje como una condición necesaria para promover cambios significativos en el aula. En este trabajo se concluyó que: "la práctica lingüística de la oralidad fundamentada en las funciones del lenguaje hace progresar al niño en sus procesos de conocimiento y lo capacita para responder a otros discursos sociales" (Jaimes Carvajal y Rodríguez Luna, 1996: 88).

En el segundo se resalta la eficacia del sistema de apoyo implementado por las docentes participantes en la experiencia, como dispositivo que posibilita el desarrollo sistemático de la competencia oral.

En el tercero se resaltó que "las estrategias discursivas del diálogo se deben construir en íntima relación con el concepto de negociación que opera tanto en el plano discursivo como en la acción social. Entender estos significados y sus beneficios para la acción humana, puede efectivamente contribuir a la generación de una cultura de la paz tan esquiva a los propósitos de construcción de nacionalidad en Colombia" (Jaimes de Casadiego, 2003).

Como se indicó, el proyecto "Cualificación de docentes y desarrollo de la argumentación en el preescolar" se propone demostrar la eficacia del sistema de apoyo del profesor para lograr desarrollos significativos de la competencia argumentativa oral en el aula de preescolar.

Esta experiencia se basó en el planteamiento de Bruner (1990) sobre la existencia de un mecanismo de adquisición que denomina Sistema de Apoyo de Adquisición del Lenguaje (LASS: Language Acquisition Support System), basado en marcos rutinarios y familiares de la comunica-

\footnotetext{
ción de la lengua materna" (Jaimes y Rodríguez, 1996) realizado con el auspicio de Colciencias y la Universidad Distrital); Proyecto Pedagógico para el desarrollo del diálogo en el preescolar". 2. "Cualificación de docentes y desarrollo de la argumentación en el preescolar" (Rodríguez, 2002), 3. "Proyecto pedagógico para el desarrollo de la oralidad en el preescolar" (Jaimes, 2004).
} 
ción ${ }^{6}$, a partir de los cuales se logra la apropiación de una lengua. En tal sentido, se propuso validar la pertinencia de un Sistema de Apoyo Pedagógico ( $S A P)$, intencionalmente utilizado por las docentes para la creación de situaciones de comunicación encaminadas al desarrollo de estrategias discursivas en el marco de acciones pedagógicas planeadas (talleres que generaran formatos de interacción, juego y conversación), con el objeto de lograr la apropiación de formas cada vez más complejas del discurso oral por parte de los niños.

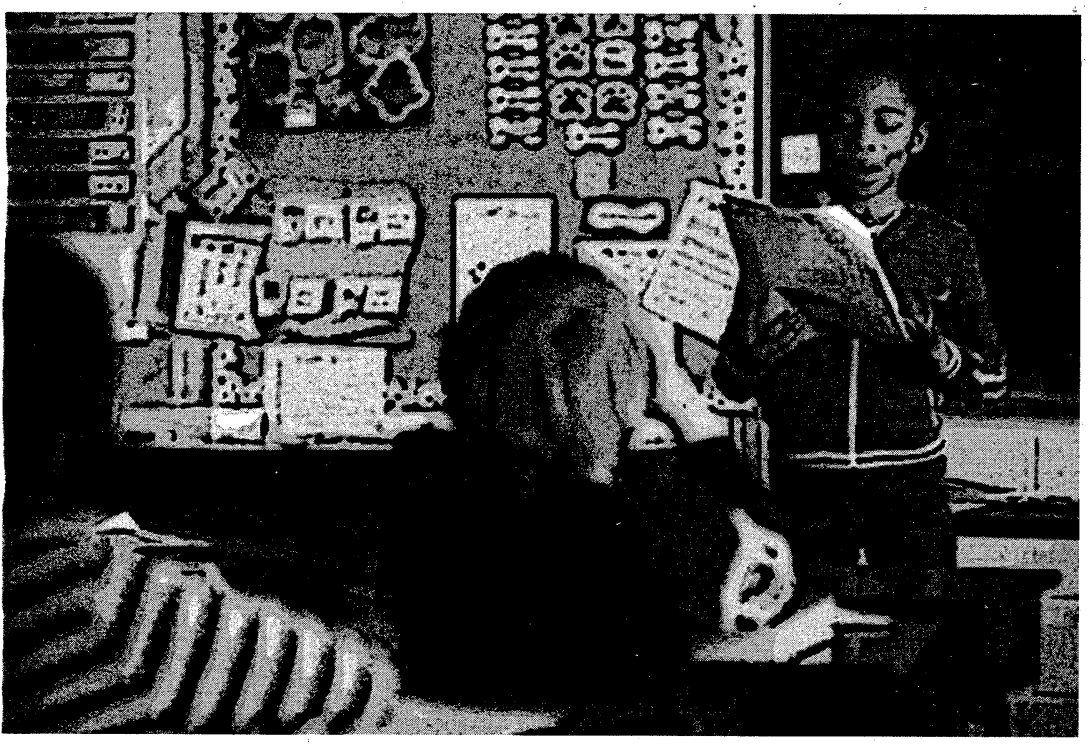

En consecuencia, el SAP se orientó a ayudar a los niños a dominar los diversos usos y funciones del lenguaje, a partir de la generación de situaciones en las cuales se requería el empleo de nuevas formas orales de la lengua para poder adecuar las estrategias de:discurso a sus intencionalidades y necesidades comunicativas, teniendo en

\footnotetext{
6 De acuerdo con Bruner, un vehículo principal del LASS es el formato. "El formato es una pauta de interacción estandarizada e inicialmente microcósmica, entre un adulto $y$ un infante, que contiene roles demarcados que finalmente se convierten en reversibles. Se convierten en rutinas tan familiares en la interacción del niño con el mundo social... modelos a partir de los cuales se construyen una interacción social y un discurso más complejos. (Ibid: 119). Los formatos al regular la interacción comunicativa antes de que comience el habla léxico-gramatical entre el niño y la persona encargada de su cuidado, constituyen unos instrumentos fundamentales en el paso de la comunicación al lenguaje." (Bruner, 1995:179).
}

cuenta los interlocutores y los contenidos que se deseaba comunicar en las interacciones.

El SAP se configuró como un escenario adecuado para el uso pertinente, contextualizado y significativo del discurso en los términos propuestos por los Lineamientos Curriculares para el Preescolar, en cuanto posibilitó la expresión de sus "conocimientos e ideas sobre las cosas, acontecimientos y fenómenos de la realidad, a construir mundos posibles, a establecer relaciones para satisfacer necesidades, formar vínculos afectivos, expresar emociones y sentimientos" (MEN, 1998b:36).

De ese modo se constató que los niños despliegan su capacidad argumentativa (considerado como uno de los discursos más complejos) ante situaciones de diálogo conducentes a la evaluación de determinados puntos de vista, la toma de decisiones respecto a un hecho o situación, la elaboración de propuestas: alternativas frente a una situación, la adopción de una posición dentro de una situación conflictiva o problemática, la expresión de la justificación respecto a la causa de un hecho o situación, la valoración del pro o el contra de una decisión o elección.

\section{Orientaciones didácticas}

A lo largo de la experiencia se constató que tales situaciones de comunicación propiciadas por los docentes con propósitos oriientados a los aprendizajes escolares fueron efectivas cuando:

- Los temas de la discusión se relacionaron con eventos cotidianos del entorno escolar, familiar o social de los niños.

- Los temas propuestos representaron para los niños situaciones interesantes frente a las cuales podían aportar sus contribuciones.

- El objeto de la discusión se encontraba ligado a una necesidad del individuo o de su grupo, es decir, cuando se trataba de resolver problemas en los cuales se hallaban directamente afectados.

- Se creó un clima de apertura hacia las diversas opiniones, se estimuló la participación a través de preguntas e intervenciones pertinentes por parte de los adultos en el sentido de promover la toma de posiciones, mantener el tema de conversación y establecer las conexiones nece- 
sarias entre los distintos puntos de vista expresados por los niños; es decir, mediante el SAP.

- Se emplearon recursos didácticos variảdos a través de los cuales se crearon situaciones adecuadas para la expresión de las opiniones de los niños.

- Se otorgó a todos el derecho a intervenir, valorando sus opiniones y respetando los turnos en la discusión, confiriéndoles el estatus de interlocutores activos a todos los miembros del colectivo sin ningún tipo de discriminación; aspecto fundamental para la construcción de la democracia.

- Se permitió la libre expresión de sus ideas sin que la censura frente a los puntos de vista divergentes limitara o coartara el contenido de sus intervenciones.

Al generar condiciones favorables para la interlocución, los docentes posibilitaron espacios de interacción de carácter funcional, contextualizado y pertinente, en los cuales los niños actuaron de manera espontánea y significativa. Estos fueron capaces de defender sus opiniones y oponerse a los argumentos de otro cuando estaban comprometidos con un tema que verdaderamente involucraba sus intereses personales.

Al mismo tiempo, los docentes apoyaron la producción argumentativa de sus alumnos, mediante la introducción de los operadores discursivos, los conectores lógicos necesarios, las precisiones conceptuales y estructuras de la lengua requeridas, haciendo uso del sistema de apoyo para lograr la apropiación del modelo de actuación sobre el cual-enfatizaron.

Aunque para los niños el dominio de la estrategia argumentativa constituye una tarea compleja por cuanto les exige tomar en cuenta y adaptarse a la perspectiva del interlocutor, deben inferir la posición de su interlocutor y los argumentos que la sostienen (cuando no son explícitos) para refutarlas al tiempo que defiende su propia perspectiva, requieren seleccionar las unidades lingüísticas apropiadas para la elaboración de tales operaciones, el uso permanente de formatos de interacción variados -en los cuales se recurrió a la argumentación en situaciones significativas para los niños-, permitió que las docentes apoyaran de forma sistemática la apropiación del discurso argumentativo.
De esta forma, les posibilitaron las condiciones básicas pasar progresivamente de formas simples y cotidianas de la argumentación -en las que también se manifiestan la justificación y la negociación para la defensa de su posición-, a discursos cada vez más complejos. Frente a ellas necesitarán, por supuesto, recurrir a nuevas estructuras lingüísticas que les permitan apropiarse del formato del discurso argumentativo of recido como modelo de interacción en el aula.

En esta experiencia se concluyó que los niños emplearon estructuras argumentativas o de orientación argumentativa cuando requirieron dar cuenta de las causas o razones que provocaron una determinada posición u opinión, gusto, preferencia, hecho o circunstancias de lugar, tiempo o modo. En tales casos, las preguntas del docente o persona que actuó como tal en la interacción, adoptaron las siguientes formas:

- Indagaron por gustos o preferencias: “¿Por qué te gusta $\mathrm{X}$ o por qué no te gusta $\mathrm{X}$ (algo o alguien)", "Por qué te gusta (Acción) o por que no te gusta $X$ (acción). Así por ejemplo, las siguientes intervenciones promovieron la construcción de estructuras argumentativas en los niños: “¿Por qué te gusta la escuela?”, “PPor qué te gustan los Simpsons?", “'Y por qué te gusta que Gokú pelee?”, “¿Por qué te gusta venir a la escuela?", “’Por qué te gustan esos programas?”

- Preguntaron las causas o razones que generaron determinadas acciones: "¿Y por qué hace paros la gente?, “¡Por qué los profesores están en paro?”, ‘’Por qué es bonito tu barrio?”, “¿Por qué sabes que robaron?” "¿Por qué era mala la princesa?, “¿Y por qué se roban los niños?”, ¿YY Willy se volvió feliz, por qué razón?”

- Solicitaron la opinión respecto a un hecho o situación: "¿Y a ustedes les parece bien eso de tirar balas?", "¿Y a ustedes les parecen bien esas peleas?”, “¿por qué tu barrio es bonito? Dime una razón", "Claro, porque se pueden herir personas, ¿cierto?", “Ah, claro, porque eso es mentira, son dibujos animados ¿no?”, "Bueno, corazón ¿te gusta la escuela?”

- Presentaron una situación conducente a una respuesta de carácter argumentativo: “¿Hay mucha inseguridad en el barrio?” 
- Solicitaron la especificación de circunstancias de lugar, tiempo o modo frente a un hecho: “¿Cuándo (ocurrió eso?)”.

La producción de estos enunciados requirió la creación de condiciones didácticas para que los niños tuvieran la oportunidad de construir argumentaciones sencillas. Los talleres y las entrevistas etnográficas constituyeron soportes importantes para este propósito, por cuanto los alumnos contaron con un espacio adecuado para la interacción, teniendo en cuenta que el SAP tuvo como finalidad específica la generación de estas construcciones. En palabras de Carsalade, Dhers y García-Debanc "los niños son extremadamente sensibles al contrato didáctico "(2001: 357) propuesto por el docente y, en consecuencia, se requiere intervenciones y actitudes adecuadas de su parte.

\section{Conclusiones}

Cierro la presente reflexión resaltando aquello que no se evalúa respecto a la oralidad en el aula y que por su importancia para el desarrollo discursivo, cognitivo y cultural de nuestros niños y jóvenes amerita ser incorporado:

a. La escucha, pues a pesar de ser la fuente de apropiación de conocimientos que más se emplea en el aula, ya que el profesor tiene el mayor tiempo el uso de la palabra, no se trabaja sistemáticamente. Se podría comenzar, por ejemplo, con la escucha de un discurso auténtico grabado con anterioridad, con el fin de analizar aspectos semánticos y pragmáticos del discurso teniendo en cuenta la interpretación del sentido que circula a partir del tono, las modulaciones; la estructura del texto, tal como efectivamente ocurre en situaciones efectivas de uso en la comunicación.

b. El análisis de la coherencia tanto para la producción como para la comprensión de textos, teniendo en cuenta que se trata de una propiedad esencial del discurso, haciendo énfasis en los géneros discursivos y los modos de organización que los caracterizan.

c. La cohesión para promover la reflexión en torno a la articulación de las diversas partes del discurso, el uso de conectores, las transiciones entre tópicos del discurso, tanto en la producción como la producción del discurso oral.

d. El uso de estrategias discursivas y el análisis de los efectos que se producen por el uso de las mismas en el auditorio, en concordancia con los propósitos de la interacción.

e. La interpretación sobre los discursos y la explicación de los fundamentos de dicha interpretación (aspectos metalingüísticos $\mathrm{y}$ metacognitivos).

f. Los modos de organización del discurso teniendo en cuenta las situaciones de comunicación (formales, no formales; cotidianas, académicas).

De otra parte, la situación actual de la educación colombiana muestra la necesidad de orientar la oralidad desde una perspectiva significativa para los estudiantes y sistemática para los docentes, a partir de acciones estratégicas como las siguientes:

a. El desarrollo de proyectos centrados en la puesta en escena de la oralidad.

' b. La incorporación del estudio sistemático de las diversas formas del discurso oral a partir de la reflexión sobre la producción oral con fundamento en las categorías teóricas propuestas en los Lineamientos y Estándares Curriculares y otras que se consideren pertinentes.

c. La definición de criterios claros para la orientación de las actividades de escuchar y hablar en el contexto escolar a partir de estrategias didácticas orientadas al desarrollo de estas competencias.

d. La construcción de instrumentos que ayuden a la interpretación de los discursos orales que circulan en nuestra cultura y a la identificación por parte de estudiantes y docentes.

e. La reflexión sobre la estructura, funcionamiento y funciones de los discursos orales cotidianos y elaborados, asumiendo las diversas competencias (gramatical, semántica, pragmática...) de modo integral.

f. La recuperación del espacio de lo oral con un estatus semejante al de la lectura y la escritura. 
g. La profundización sobre la naturaleza de la oralidad, sus formas, géneros y demás aspectos pertinentes, a partir de la investigación en el aula.

h. El reconocimiento de la oralidad como espacio adecuado para la construcción de la alteridad, el reconocimiento del otro, la aceptación de las diferencias, el debate y la crítica.

i. El desarrollo de actividades en el aula orientadas al uso de la argumentación, la ex- . plicación, el diálogo y la narración en situaciones reales de uso.

j. El fortalecimiento de la reflexión sobre el sentido que se construye a través de la oralidad y su incidencia en las formas de pensar, ser y vivir la cultura.

Esto implica, por supuesto, desarrollar investigación sobre el desarrollo de la oralidad en el aula. Interrogantes como los siguientes podrían ser objeto de trabajo en los colectivos y redes:

1. ¿De qué modo lo oral puede constituirse en un sistema de apoyo para el desarrollo de la lectura y la escritura?

2. ¿Qué tipo de actividades se proponen en el aula para el desarrollo de la oralidad?

3. ¿Qué avances se logran en el dominio de lo oral a partir de la realización de proyectos centrados en este propósito?

4. ¿Qué relación existe entre las actividades orales y los desempeños en la eșcritura y la lectura?

5. ¿Cuál es el papel de los géneros discursivos orales en los aprendizajes escolares?

6. ¿Qué fundamentos disciplinares se requieren para abordar la oralidad en el aula?

7. ¿De qué modo estos fundamentos se pueden tener en cuenta para la evaluación de lo oral?

8. ¿Qué condiciones didácticas se requiere generar para favorecer el desarrollo de la oralidad en el aula?

9. ¿Qué criterios se podrían considerar para sistematizar la evaluación de la oralidad en el aula?

E1 desarrollo de la oralidad en el aula involucra el esfuerzo de profesores e investigadores para profundizar en un campo del lenguaje que requiere fortalecerse para promover logros efectivos en la interacción. Ó

\section{Bibliografía}

ABASCAL, Dolores (1993). "La enseñanza oral en la enseñanza secundaria". En: Lomas Carlos: El enfoque comunicativo en la enseñanza de la lengua. Paidós, Barcelona.

ATORRESI, Ana (2005). Material del Seminario Evaluación Externa y evaluación de ítemes. Icfes - Universidad Nacional. Bogotá.

BENVENISTE, Claire Blanche (1998). Estudios lingüisticos sobre la relación entre oralidad y escritura. Gedisa. Barcelona.

BRUNER, Jerome (1995). Acción, pensamiento y lenguaje. Alianza Psicología: Madrid.

BRUNER, Jerome y Haste, Helen (1990). La elaboración del sentido. Paidós: Barcelona.

CARSALADE, Pascales, Dhers, Marivone y García-Debanc, Claudine (2001). "Les conduites discursives d'argumentation ou quand discuter aide à apprendre les sciences. En: Loral dans la classe. Discours, métadiscours, interactions verbales et construction de savoirs à l'école primaire. Coordonné par Michel. Grandaty et Gilbert Turco. INRP, Paris.

CALSAMIGLIA Blancafort, Helena y Tusón Valls, Amparo (1999). Las cosas del decir. Manual de análisis del discurso. Ariel Lingüística: Barcelona.

Colegiatura de lenguaje del Icfes (2005). Documentos de trabajo. Boletín No. 1.

CORREA, José Ignacio, Dimaté, Cecilia y otros (2003): Estándares de lenguaje (Lengua Castellana, literatura y otros sistemas simbólicos). Documento de trabajo no publicado.

HALLIDAY, M.A.K. (1986) El lenguaje como semiótica social. Fondo de Cultura Económica: Méjico.

HYMES, Dell. (1972). “Acerca de la competencia comunicativa”. En: Pride and Holmes (eds). Sociolinguistics, 1972.pp. 269-293. Traducido por Juan M. Gómez B. p. 32 (Mimeo).

JAIMES CARVAJAL, Gladys y Rodríguez Luna, María Elvira (1996). Lenguaje y mundos posibles. Una propuesta para la Educación Preescolar. Universidad Distrital - Colciencias, Bogotá. 
JAIMES DE CASADIEGO, Gladys (2003) "Diálogo, dialogismo e interlocución" En: Revista Enunciación No. 8 (pp. 20-27).

LOMAS, Carlos (1999). Cómo eñ̀señar a bacer cosas con las palabras. Vol. 2 Paidós, Barcelona.

MEN (1998a). Lineamentos curriculares en lengua castellana. Editorial Magisterio: Bogotá.

MEN (1998b). Lineamientos pedáógicos para la educación formal. Preescolar. Serie. Lineamientos Curriculares: Bogotá.

OLSON, David (1995). Cultura escrita y oralidad. Gedisa, Barcelona.

ONG, Walter (1987). Oralidad y escritura. Fondo de Cultura Económica: Méjico.
REYZÁBAL, María Victoria (1993). La comunicación oral y su didáctica. Editorial La Muralla S.A.: Madrid.

RODRÍGUEZ LUNA, María Elvira (2003). Formación, interacción, argumentación. Fondo de publicaciones Universidad Distrital: Bogotá. . (2005) "La evaluación externa y la elaboración de ítemes. Algunas consideraciones sobre la construcción de pruebas". En: Revista Enunciación No. 10. Fondo de Publicaciones Universidad Distrital Francisco José de Caldas: Bogotá. pp. 133-139. 